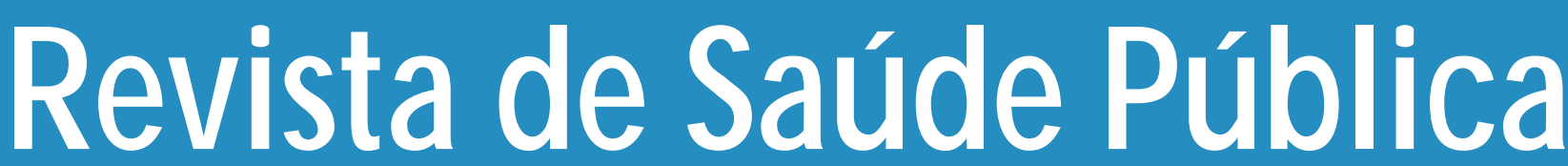

Marcação-soltura-recaptura para determinar o tamanho da população natural de Anopheles albitarsis l.s. (D iptera: Culicidae) M arking-release-recapture methods for determining the size of the natural population of Anopheles albitarsis I.s. (Diptera: Culicidae)

Roseli La Corte dos Santos e 0 swaldo Paulo Forattini

Departamento de Epidemiologia da Faculdade de Saúde Pública da Universidade de São Paulo. São Paulo, SP - Brasil 


\title{
Marcação-soltura-recaptura para determinar 0 tamanho da população natural de Anopheles albitarsis l.s. (Diptera: Culicidae)*
}

\section{Marking-release-recapture methods for determining the size of the natural population of Anopheles albitarsis I.s. (Diptera: Culicidae)}

\author{
Roseli La Corte dos Santos e O swaldo Paulo Forattini \\ Departamento de Epidemiologia da Faculdade de Saúde Pública da Universidade de São Paulo. São \\ Paulo, SP - Brasil
}

\section{D escritores}

Anopheles.

Ecologia de vetores.

\section{Resumo}

Objetivo

Objetivou-se estimar o tamanho de população natural de Anopheles albitarsis s.l. presente em fazenda de plantação com arroz irrigado no Vale do Ribeira, SP, Brasil, no período do verão, como subsídio para avaliação da capacidade vetora.

\section{Métodos}

Foram feitos três experimentos de marcação-soltura-recaptura com pó fluorescente, com populações de campo e populações criadas em laboratório. Foram realizadas, concomitantemente, capturas com isca humana.

\section{Resultados}

A população estimada em três eventos de soltura foi $64.560,50.503$ e 22.684 mosquitos. A taxa de picadas/homem/noite variou entre 41,5 e 524,5.

\section{Conclusão}

Observou-se alta densidade de mosquitos no período considerado, permitindo inferir que, ainda que a sobrevivência da espécie seja baixa, número substancial de fêmeas pode sobreviver tempo suficiente para ultrapassar o período extrínseco de desenvolvimento do parasita.

\section{Abstract}

Anopheles.

Ecology, vectors.

\section{O bjective}

In order to evaluate the size of the Anopheles albitarsis s.l. population in the Ribeira Valley region of the south-eastern S. Paulo State, Brazil, marking-release-recapture experiments were carried out on the Experimental Farm in Pariquera-Açu county where an artificial irrigation system for the cultivation of rice has been installed. The experiments aimed at determining the vectorial capacity of this species.
Correspondência para/Correspondence to: Roseli La Corte dos Santos Av. Dr. Arnaldo, 715 01246-904 São Paulo, SP-Brasil E-mail: rlacorte@usp.br
*Pesquisa financiada pela Fundação de Amparo à Pesquisa do Estado de São Paulo (FAPESP) (Projeto Temático 95/0381-4, Bolsa DR-2 96/9174-4). Realizada no Núcleo de Pesquisa Toxonômica e Sistemática em Entomologia Médica (NUPTEM). Edição subvencionada pela FAPESP (Processo n 98/13915-5). Recebido em 1.9.1998. Aprovado em 2.12.1998. 


\section{Methods}

During the summer (January, February and March), three marking-release-recapture experiments with field and laboratory reared populations were conducted to evaluate the population size. Simultaneous human bait collections were also carried out to estimate the human biting rate.

\section{Results}

The population sizes of the three experiments were estimated to be 64,560, 50,503 and 22,684 mosquitoes. The bite/man/night rate varied from 41.5 to 524,5 .

\section{Discussion}

The results indicated high mosquito density in the summer which allow one to infer that, even though the survival rate is low, the number of remaining females is large enough to outlive the parasite's extrinsic developmental period.

\section{INTRO DUÇÃO}

A marcação-soltura-recaptura constitui método adaptado para a finalidade de obter informações que permitam a estimativa da densidade e da sobrevivência de populações animais. Em epidemiologia, essa metodologia tem sido aplicada a populações de vetores biológicos de agentes infecciosos, como subsídio para a avaliação da capacidade vetora.

O papel do Anopheles albitarsis como transmissor não tem sido considerado de maneira constante. Segundo Rachou? (1958), esta espécie participaria como vetor principal ou auxiliar na transmissão de malária, estando a capacidade vetora ligada principalmente à sua densidade e freqüência nos domicílios. Na região do Vale do Ribeira, têm sido desenvolvidas pesquisas com a finalidade de conseguir informações sobre o comportamento da fauna de mosquitos Culicidae. Alocalidade representada pela $\mathrm{Fa}$ zenda Experimental do Instituto Agronômico em Pariquera-Açu foi uma das escolhidas e ali pôde-se observar a associação desse anofelíneo com o sistema de cultivo irrigado de arroz (Forattini et al. ${ }^{2,3}$, 1993). Nessa ocasião, considerou-se a população local do mosquito como eusinantrópica, o que levantou a necessidade de se estimar a capacidade vetora de An. albitarsis uma vez que este anofelíneo cresce em importância na medida em que vão sendo implantadas condições que lhes são favoráveis ao desenvolvimento. Tal seria o caso do sistema de irrigação artificial na prática agrícola, propiciando-lhe contato mais freqüente com o homem.

O presente trabalho teve por objetivo estimar o tamanho populacional do mosquito na citada localidade e no período do verão, visando a avaliar sua capacidade vetora.

\section{MÉTODOS}

A área estudada localiza-se na Fazenda Experimental do Instituto Agronômico de Campinas, no Município de Pariquera-Açu, Estado de São Paulo. Desenvolve-se ali plantação de arroz irrigada artificialmente. Descrição pormenorizada da área pode ser encontrada em Forattini et al. ${ }^{2}$ (1993). Ao redor da plantação de arroz encontram-se manchas de mata residual, as quais também já foram alvo de estudos anteriores (Forattini et al. ${ }^{3}, 1993$ ).

\section{Marcação}

Adotaram-se dois tipos de procedimentos. Um deles constituiu-se da utilização de espécimes criados em laboratório, posteriormente marcados e soltos. $\mathrm{O}$ outro foi o de usar fêmeas coletadas no campo. Para obter indivíduos F1, procedeu-se à coleta de fêmeas, as quais foram alimentadas com sangue e mantidas individualmente em borréis para oviposição. As larvas foram criadas segundo a orientação de Horosko III et al. ${ }^{5}$ (1997), com ração para peixe, TetraMin ${ }^{\circledR} 5$ Star e TetraMin ${ }^{\circledR}$ "baby fish food" "E", e apresentaram tempo de desenvolvimento médio de 10 dias até a fase pupal. A maioria das larvas foram criadas em lotes de 100 exemplares e apresentaram mortalidade média de $24 \%$. Na medida em que se formavam, as pupas eram transferidas para frascos menores e mantidas em gaiolas de "nylon". Após a eclosão, as exsúvias das pupas eram retiradas e utilizadas para determinação do número de fêmeas presentes. A marcação dos adultos foi realizada com pó fluorescente, utilizando insuflador. Logo após a marcação, os mosquitos eram levados até o local escolhido e libertados. Os mosquitos para soltura direta foram coletados em armadilha tipo Shannon, operada por duas pessoas. Foi utilizado aspirador manual, adaptado com tubo de vidro na extremidade para diminuir e direcionar o campo de sucção, de modo a permitir a captura individual e, portanto, a contagem dos mosquitos enquanto eles eram capturados. Quando era atingido o número de 30 mosquitos, o conteúdo do aspirador era transferido para as gaiolas. Foram recolhidas amostras de cada pessoa com a finalidade de determinar possível erro de contagem e percentual de outras espécies de Anopheles, passíveis de confusão. Os mosquitos foram levados ao la- 
boratório, onde passavam a noite. Foi-lhes fornecida solução de sacarose como alimento e colocada toalha úmida sobre a gaiola. No dia seguinte, o alimento era retirado e os mosquitos marcados e libertados.

Com esse procedimento, foram soltos três lotes de mosquitos: o primeiro em 1/2/98 composto por 475 fêmeas de até 24 h de vida; o segundo em 3/2/98 com 315 fêmeas de até 45 h de vida; e o terceiro em 9/3/98 com 431 fêmeas de campo com idade indeterminada. Os adultos foram soltos às $4 \mathrm{~h}$ da tarde, em duas direções diferentes, à margem das quadras de arroz onde se supunha estarem localizados os criadouros. Os pontos de soltura distavam cerca de $25 \mathrm{~m}$ do local escolhido para recaptura. $\mathrm{O}$ afastamento do ponto de recaptura e a soltura à tarde, em horário anterior ao de pico de atividade, visaram a garantir a mistura dos mosquitos marcados com aqueles presentes no campo. As recapturas realizaram-se no mesmo dia da soltura, com a utilização de armadilha de Shannon, em posição média em relação aos dois pontos de soltura. As capturas estenderam-se por 40 min durante o período do crepúsculo, com auxílio de aspirador manual. Os mosquitos capturados foram levados ao laboratório, examinados sob luz ultravioleta, em câmara escura, contados e identificados. A probabilidade de recaptura foi considerada igual para mosquitos marcados e não marcados.

\section{Isca Humana}

Os mosquitos adultos foram coletados regularmente, com freqüência quinzenal. Dois coletores foram utilizados como isca humana os quais capturavam, com aspirador manual, os mosquitos que se aproximavam para sugar. As capturas foram realizadas durante o período do crepúsculo com o início variando entre 17:40h e 18:21h e o fim variando entre 19:52h e 20:49h, segundo os horários fornecidos pelo Almanaque Náutico da Marinha do Brasil.

\section{Capacidade Vetora}

A Capacidade Vetora ( $\underline{\mathrm{C}}$ ) foi definida por GarrettJones $^{4}$ (1964) como a taxa diária de contatos potencialmente infectantes, através da população vetora:

$$
C=\frac{m a^{2} p^{n}}{-\log (p)}
$$

onde:

$m=$ densidade do mosquito em relação ao homem;

$a=$ número médio de picadas/ fêmea/ dia;

$p=$ probabilidade de sobrevivência diária do mosquito;

$n=$ duração do ciclo extrínseco do parasita.
O presente trabalho não objetivou o cálculo da capacidade vetora "stricto sensu", mas sim contribuir com a estimativa dos parâmetros $m$ e $a$. Uma vez que o produto ma representa a taxa de picadas por homem por noite (Reisen $\left.{ }^{10}, 1989\right)$, ele pode ser obtido diretamente do valor encontrado com a realização coletas com isca humana e, desde que conhecido um dos dois parâmetros, pode ser calculado o segundo. Assim, descobrindo-se o valor do produto $m a$ e a estimativa do tamanho da população $m$, pode-se obter expectativa do comprimento do ciclo gonotrófico através de $a$, supondo-se ocorrência de concordância gonotrófica.

\section{Tamanho Populacional}

Para o cálculo do tamanho da população (P) foi utilizado o índice de Lincoln corrigido por Bailey ${ }^{1}$ (1952) para amostras de pequeno tamanho $(r<20)$. Trata-se de modelo determinístico no qual a estimativa de $\mathrm{P}$ é obtida através de único evento de marcação:

$$
P=\frac{a(n+1)}{r+1}
$$

com variância:

$$
\mathrm{V}=\frac{a^{2}(n+1)(n-r)}{(r+1)^{2}(r+2)}
$$

onde:

$a=$ número de mosquitos marcados;

$n=$ número total de mosquitos na segunda amostra;

$r=$ número de mosquitos marcados na segunda amostra.

\section{RESULTAD OS}

As três capturas realizadas com armadilha de Shannon renderam o total de 4.539 mosquitos entre marcados e não marcados. Do total de 1.221 mosquitos marcados e soltos, 38 foram recapturados (Tabela). A taxa de recaptura foi de $2 \%$ em 1/2, $3 \%$ em $3 / 2$ e $4 \%$ em $9 / 3$.

O tamanho da população (P), calculado para os três eventos de soltura foi: 64.560 em 1/2, 50.503 em $3 / 2$ e 22.684 em $9 / 3$. Com isca humana foram capturados 3.414 mosquitos durante o período (Tabela 2). A quantidade de mosquitos foi aumentando

Tabela 1 - Estimativa do tamanho da população de Anopheles albitarsis l.s. na Fazenda Experimental, durante o verão, calculado através do índice de Lincoln modificado por Bailey. Pariquera-Açu, SP, 1998.

\begin{tabular}{lcccccc}
\hline Data & Soltos(a) & Recap.(r) & Não marcados & Total(n) & População(P) & Desvio-padrão \\
\hline $1 / 2 / 98$ & 475 & 11 & 1.631 & 1.642 & 64.560 & \pm 17.972 \\
$3 / 2 / 98$ & 315 & 09 & 1.889 & 1.898 & 59.503 & \pm 17.988 \\
9/3/98 & 431 & 18 & 981 & 999 & 22.684 & \pm 5.024 \\
\hline Total & 1.102 & 38 & 4.501 & 4.539 & & \\
\hline
\end{tabular}


no mês de janeiro, apresentando pico no dia 4 de fevereiro e diminuindo em seguida. O número de picadas/homem/noite ( $m a$ ) variou entre 41,5 e 524,5.

$\mathrm{O}$ valor estimado para $a$ foi 0,01 picadas/fêmea/ noite quando consideradas as populações de 3/2 e 9/3 e os valores de ma com datas mais próximas a essas.

A Figura traz as medidas pontuais do tamanho da população e os resultados das capturas quinzenais com isca humana realizadas durante o mesmo período.
Tabela 2 - Distribuição do número de Anopheles albitarsis I.s. capturados em isca humana na Fazenda Experimental, durante o verão. Pariquera-Açu, SP, 1998.

\begin{tabular}{rcc}
\hline \multicolumn{1}{c}{ Data } & $\begin{array}{c}\mathrm{N} \underline{0} \\
\text { capturado }\end{array}$ & $\begin{array}{c}\mathrm{N} \underline{\mathrm{O}} \\
\text { picadas/homem/noite(ma) }\end{array}$ \\
\hline $8 / 1 / 98$ & 340 & 170 \\
$21 / 1 / 98$ & 614 & 307 \\
$4 / 2 / 98$ & 1.049 & 524,5 \\
$18 / 2 / 98$ & 779 & 389,5 \\
$4 / 3 / 98$ & 549 & 274,5 \\
$18 / 3 / 98$ & 83 & 41,5 \\
\hline Total & 3.414 & 1.707 \\
\hline
\end{tabular}

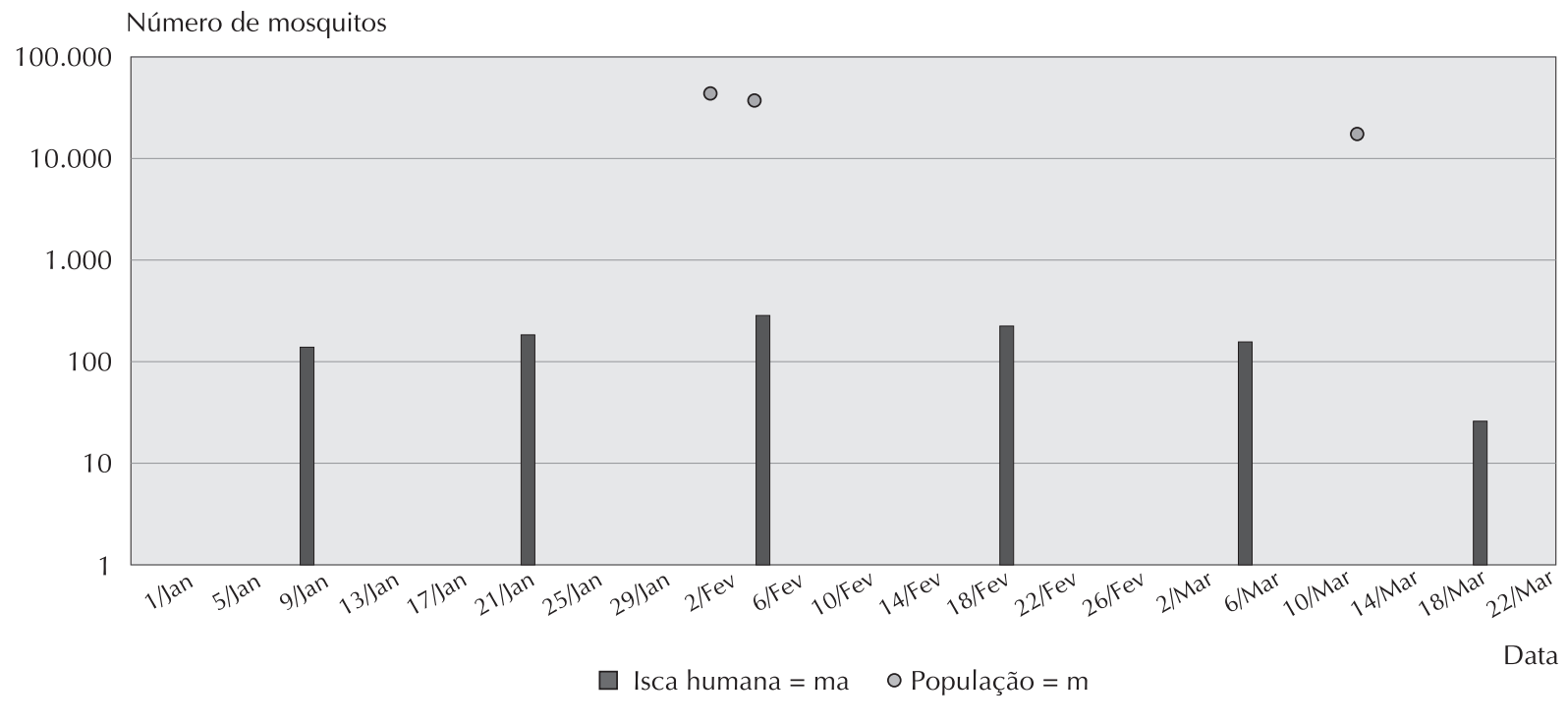

Figura - N úmero de Anopheles albitarsis s.l. capturados quinzenalmente com isca humana e medidas pontuais do tamanho populacional, durante o período do verão de 1998, na Fazenda Experimental, Pariquera-Açu, SP.

\section{DISCUSSÃO}

As suposições básicas para utilização do método de marcação-soltura-recaptura, com o objetivo de calcular o tamanho da população são: 1- os mosquitos não devem ser afetados pela marcação; 2- os mosquitos devem misturar-se completamente com a população de campo; 3 - a probabilidade de se capturar o espécime marcado é a mesma que capturar o não marcado; 4- a população deve poder ser considerada fechada, com pequeno movimento entre as populações locais (Reisen ${ }^{8}$ 1978, Touré et al. ${ }^{12}$, 1998). Foram realizados ensaios prévios e não se detectou diferença na taxa de mortalidade entre mosquitos marcados e não marcados, permitindo supor que a marcação não estaria afetando a sobrevivência (com taxa de mortalidade igual a 0,396 \pm 0,027 1/dia para o grupo-controle e $0,381 \pm 0,015$ 1/dia para o grupo-marcado). Para permitir que ocorresse mistura, os culicídeos foram soltos a, aproximadamente, $25 \mathrm{~m}$ do local de recaptura e 2:30 $\mathrm{h}$ antes do horário de dispersão dos mosquitos. $\mathrm{O}$ isolamento de An. albitarsis l.s. na Fazenda Experimental já foi registrada por Forattini et al. ${ }^{3}$ (1993) que, tendo realizado capturas na mata residual no entorno da Fazenda, não detectaram a presença significativa desta espécie. A probabilidade de recaptura foi considerada igual para espécimes marcados e não marcados.

A presente pesquisa teve a finalidade de ajudar a compreender melhor a dinâmica da população de $A n$. albitarsis l.s. e, assim, contribuir para o conhecimento de sua capacidade vetora local. A sazonalidade, associada ao ritmo do plantio de arroz (Forattini et al. ${ }^{2}$, 1993) parece ser peça chave com relação à dinâmica populacional desta espécie. $\mathrm{O}$ ambiente altamente favorável permite que a população possa alcançar cifras como as expostas nesta observação. A taxa de recaptura, embora baixa, é compatível com a obtida 
em trabalhos semelhantes com outras espécies de anofelíneos (Reisen et al. ${ }^{9}, 1979$, Thomson et al. ${ }^{11}$, 1995, Quiñones et al. $\left.{ }^{6}, 1997\right)$, mesmo porque, nesse caso, a taxa refere-se a único evento de recaptura, conforme o previsto pelo modelo utilizado. O tamanho elevado da população vai ao encontro das afirmações de Rachou (1958) de que An. albitarsis pode ser espécie de elevada densidade. Todavia é recomendável a realização de estudos que levem em consideração a taxa de sobrevivência, objetivando conhecer a fração dessa população que sobrevive para ultrapassar o ciclo extrínseco do parasita. Os resultados aqui encontrados indicam que, devido ao tamanho da população nessa época do ano, mesmo que a probabilidade de sobrevivência seja baixa, ainda assim a transmissão poderia ocorrer. Se nessa época do ano a taxa de sobrevivência diária fosse, por exemplo, 0,5 , considerandose um ciclo extrínseco de 8 dias para Plasmodium vivax, e uma população de aproximadamente 50.000 mosquitos, como foi a de fevereiro, provavelmente 195 fêmeas sobreviveriam tempo suficiente para se tornarem infectantes. Reisen et al. ${ }^{9}$ (1979) utilizaram cálculos semelhantes, a partir dos resultados de tamanho da população e sobrevivência, para concluir que Anopheles subpictus não desempenhava papel importante na transmissão de plamódio humano no Paquistão, uma vez que, de acordo com seus resultados, apenas 1,2 fêmeas provavelmente sobreviveriam para ultrapassar a duração do ciclo extrínseco do parasita.

\section{REFERÊNCIAS}

1. Bailey NTJ. Improvements in the interpretation of recapture data. JAnim Ecol 1952; 21:120-7.

2. Forattini OP, Kakitani I, Massad E, Marucci D. Studies on mosquitoes (Diptera: Culicidae) and anthropic environment. 2. Immature stages research at a rice irrigation system location in South-Eastern, Brazil. Rev Saúde Pública 1993; 27:227-36

3. Forattini OP, Kakitani I, Massad E, Marucci D. Studies on mosquitoes (Diptera: Culicidae) an anthropic environment. 3. Survey of adults stages at the rice irrigation system and the emergence of Anopheles albitarsis in South-Eastern, Brazil. Rev Saúde Pública 1993; 27:313-25.

4. Garrett-Jones C. Prognosis for the interruption of malaria transmission through assessment of the mosquito's vectorial capacity. Nature 1964; 204:1173-5.

5. Horosko III S, Lima JBP, Brandolini MB. Establishment of free-mating colony of Anopheles albitarsis from Brazil. $J$ Am Mosq Control Assoc 1997; 13:95-6.

6. Quiñones ML, Lines JD, Thompson MC, Jaware M, Morris J, Greenwood BM. Anopheles gambiae gonotrophic cycle duration, bitin and exiting behavior unaffected by permethrin-impregnated in the Gambia. Med Vet Entomol 1997; 11:71-8
Com relação à expectativa de obter o comprimento do ciclo gonotrófico através da substituição de $m$ na taxa de picadas $m a$, nota-se que o valor obtido não é compatível com a realidade. A estimativa para $a$, assim calculada, não é indicativa do comprimento do ciclo gonotrófico, porque se o número médio de picadas por fêmea, por dia, é 0,01 , cada fêmea só voltaria a picar a cada 100 dias. Os valores de $m a$ aqui apresentados referem-se à captura de mosquitos que se aproximavam para picar e, portanto, podem mostrar-se elevados em relação aos obtidos em pesquisas cuja técnica consistiu em capturar espécimes no ato do repasto. A discrepância observada entre o tamanho da população e a quantidade capturada em isca humana sugerem:

a - população muito jovem, ainda não apta a picar, compareceria na armadilha de Shannon, mas não em isca humana;

b - baixa antropofilia. Neste caso, o valor obtido para a, pela substituição de $m$ no produto $m a$ ("man biting rate"), não é indicador do intervalo entre as picadas;

c- viés introduzido pela técnica empregada. Isso porque as pessoas não seriam capazes de capturar todos os mosquitos que se apresentam para picar, ou o raio de atração em isca humana é menor que o do em armadilha de Shannon.

7. Rachou RG. Anofelinos do Brasil: comportamento das espécies vetoras de malária. Rev Bras Malariol Doenças Trop 1958; 10:145-81.

8. Reisen WK, Aslam Y, Siddiqui TF, Khan AQ. A markrelease-recapture experiment with Culex tritaeniorhynchus Giles. Trans R Soc Trop Med Hyg 1978; 72:167-77.

9. Reisen WK, Mahmood F, Parveen T. Anopheles subpictus Grassi: observations on survivorship and population size using mark-release-recapture and dissection methods. Res Popul Ecol 1979; 21:12-29.

10. Reisen WK. Estimation of vectorial capacity: Introdution. Bull Soc Vector Ecol 1989; 14:39-40.

11. Thompson MC, Connor SJ, Quiñones ML, Jawara M, Todd J, Greenwood BM. Movement of Anopheles gambiae s.l. malaria vectors between villages in the Gambia. Med Vet Entomol 1995; 9: 413-9.

12. Touré YT, Dolo G, Petrarca V, Traoré SF, Bouaré M, Dao A, Carnahan J, Taylor CE. Mark-release-recapture experiments with Anopheles gambiae s.l. in Banambani Village, Mali, to determine population size and structure. Med Vet Entomol 1998; 12:74-83. 